\title{
Blood pressure and pulse wave velocity measurement for operant conditioning of autonomic responding*
}

\author{
G. C. JERNSTEDT \\ Dartmouth College, Hanover, New Hampshire 03755 \\ and \\ JEFFREY P. NEWCOMER \\ School of Medicine, Boston University, Boston, Massachusetts 02215
}

\begin{abstract}
Presently available procedures for measuring blood pressure are not completely satisfactory for Es wishing to investigate the operant conditioning of cardiovascular activity. A system is described for measuring a correlate of the blood pressure information obtained with conventional sphygmomanometric systems. The procedure involves the measurement of speed of propagation through the arterial system of the pressure pulse from the heart's contraction. This speed, the pulse wave velocity, is closely related to cuff-derived blood pressure. Many of the problems present with current sphygmomanometric systems are absent with this apparatus and procedure. The apparatus can be assembled from common instruments or constructed from integrated circuits. Data collected with the apparatus confirm earlier studies which indicate that pulse wave velocity is correlated with sphygmomanometrically measured blood pressure and is a valuable cardiovascular response measure.
\end{abstract}

Operant conditioning techniques have been employed to train animals to effect changes in a great variety of responses mediated by the autonomic nervous system (cf. Miller \& Banuazizi, 1968). Operant techniques have also been used successfully in controlling autonomic responses with human Ss, particularly responses of the cardiovascular system (cf. Shapiro, Tursky, Gershon, \& Stern, 1969). Perhaps the most interesting property of the cardiovascular system that has been controlled in humans is blood pressure, because of the complexity of the bodily responses involved and because of the great importance of blood pressure to the health of the organism. Current evidence suggests that some changes in specific autonomic activity which have been observed in cardiovascular conditioning are not due to generalized autonomic activity resulting from skeletal or cognitive mediation (Miller \& Banuazizi, 1968). In particular, with human Ss, Shapiro et al (1969) were able to condition changes in blood pressure independent of changes in heart rate, and Shapiro, Tursky, and Schwartz (1970b) succeeded in conditioning changes in heart rate which were independent of changes in blood pressure.

A major obstacle to the investigation of the operant conditioning of cardiovascular responses has been the difficulty of measuring response occurrence. During operant conditioning of skeletal responses, feedback to the $\mathrm{S}$ and $\mathrm{E}$ is usually provided by a simple manipulandum. In the conditioning of autonomic responses, however, a much more sophisticated apparatus is needed.

Ideally, a response measurement device should

*This research was supported in part by Grant 2T01 HEO 5303-13 from the National Heart Institute. provide information about a response: (a) immediately after the response, (b) continuously over long periods of time without necessary interruption, (c) with allowance for $E$ control of feedback to the $S$ about the response and reinforcement, (d) reliably and simply, (e) without alteration of the response being measured, and (f) if possible, with allowance for free movement of the $S$ in his environment. None of the devices currently used to monitor blood pressure in human Ss satisfies more than two of the above criteria, and most satisfy only one. This paper presents a system for the precise measurement of a correlate of blood pressure that can satisfy all six of the above criteria.

\section{METHOD}

\section{Principles}

During the cardiac cycle, the contractions of the left ventricle, which eject the blood from the heart into the arterial system, impart a velocity to the blood, forcing it through the arteries, and produce a pressure wave which moves along the elastic arteries. It is this pressure wave and not the flow of blood that is felt as the pulse beat.

Most of the measuring devices used in experiments on the operant control of blood pressure in humans have been variations of the basic sphygmomanometer (the arm cuff and hand pump used by most physicians). This device employs a calibrated external pressure on the wall of an artery and measures the point at which the external pressure prevents the pulse wave from passing through the artery. Shapiro et al (1969) employed an arm cuff that was automatically inflated and deflated by a pneumatic system. A sensitive microphone was used to detect the Korotkoff sounds, and blood pressure was assumed to be equal to that external pressure on the cuff at which Korotkoff sounds were heard for $50 \%$ of the heart pulses. Brener and Kleinman (1970) used a device which operated in the same way as that used by Shapiro et al (1969), but which 


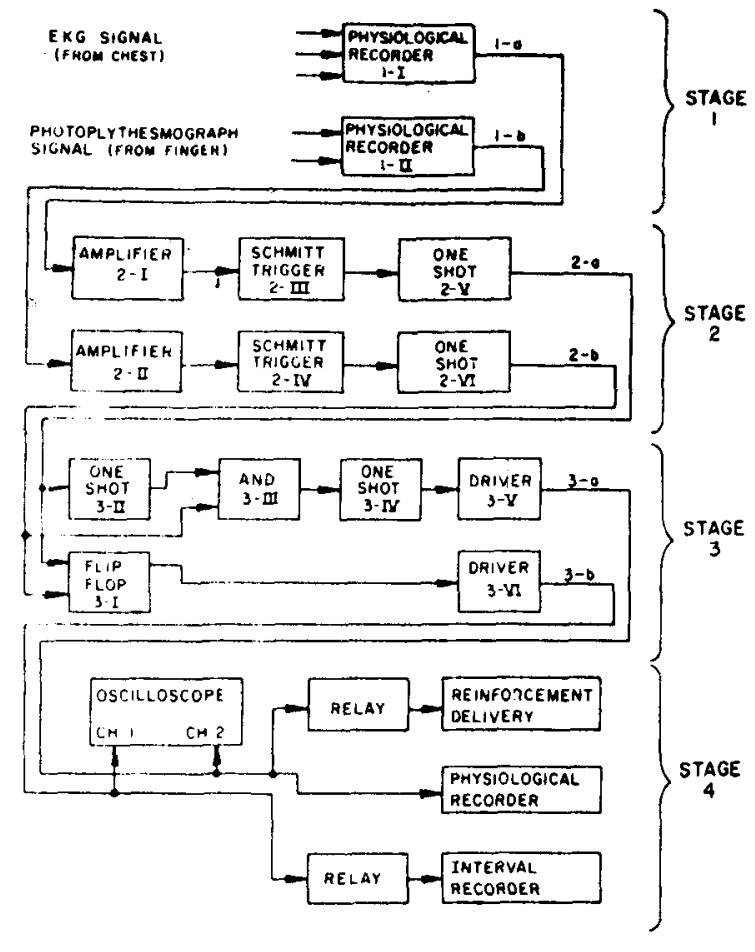

Fig. 1. Overall block diagram of the measurement apparatus.

employed a cuff around the S's finger.

Unfortunately, there are inherent limitations in the sphygmomanometric methods of blood pressure measurement. Since the cuff occludes the blood vessels in the limb to which it is attached, it cannot be used for more than a short period of time before it must be deflated to allow normal blood flow (Shapiro et al, 1969). Brener and Kleinman (1970) adjusted the finger cuff to allow venous return, and so periodic deflation was avoided. But both arm and finger-cuff methods required the repeated occlusion of blood vessels, which can affect the blood pressure being measured. It has been suggested that even with periodic release of the occluding pressure there is a risk of physical trauma, especially over the long periods of conditioning that may be required for therapeutic blood pressure modification (Shapiro et al, 1970a). A deficiency with both the arm and the finger-cuff methods is that rather than providing a continuous measure of blood pressure, these methods only indicate whether the blood pressure is above or below the criterion pressure exerted by the cuff. A further problem with the cuff method is that it produces skin sensations on the arm or finger which provide the $S$ with sensory feedback about whether he is above or below criterion. The availability of this feedback to the $\mathrm{S}$ cannot be controlled by the E. Finally, cuff methods require a rather complicated pneumatic inflation and deflation system, thus limiting their simplicity and portability. These problems have led to the present investigation of pulse wave velocity as a correlate of arterial blood pressure.

Weltman, Sullivan, and Bredon (1964) reported that pulse-wave velocity can be expressed mathematically when certain simplifying assumptions are made about the cardiovascular system. They concluded that pulse wave velocity varies inversely with the square root of arterial extensibility, and that extensibility in humans varies with age, pathological condition, and arterial pressure. Similar conclusions have been discussed and experimentally examined by others (cf. Williams \& Williams, 1965). At any one point in time and for any one individual, both age and pathology remain fixed, so pulse wave velocity and arterial pressure should be directly related. Thus, for Ss in operant conditioning experiments, pulse wave velocity and conventionally measured systolic or diastolic pressure are both functions of certain parameters of cardiovascular activity which have been shown to contribute to mean arterial pressure.

In fact, correlations between blood pressure and pulse wave velocity within Ss are typically quite high. For example, Sands (1925) reported a correlation between diastolic blood pressure and pulse wave velocity of 0.72 , and between systolic blood pressure and pulse wave velocity of 0.82 . King (1950), in reviewing literature on pulse wave velocity, presented data showing a regular, direct relationship between mean blood pressure and pulse wave velocity. Considering the degree of error in the measurement of blood pressure by cuff methods $(6-8 \mathrm{~mm}$ at best, as reported by Smith \& Bickley, 1964), one might conclude that if the cuff methods were more accurate, an even higher correlation between blood pressure and pulse wave velocity should be observed.

\section{Procedure}

The procedure described here reflects blood pressure changes by measuring pulse wave velocity changes. Pulse wave velocity is determined by measuring the lapsed interval between the major (R) wave of the electrocardiogram (EKG) and the arrival at a fixed location on the index finger of the pressure pulse created by that contraction of the heart. The arrival of the pulse wave at the finger is detected by recording the output of a photoplethysmograph. Using only these two events, one can obtain information about a number of parameters of the cardiovascular system, for each beat of the heart. Heart rate can be calculated by measuring the interval of time between two successive $R$ waves. Pulse transit time can be calculated by measuring the interval of time between the EKG pulse and the photoplethysmograph pulse. Pulse wave velocity can be obtained by dividing the arterial distance from the heart to the location of the photoplethysmograph by the pulse transit time. Normally, the operant investigator would not be interested in pulse wave velocity, but would be interested only in pulse transit time. For constant placements of the photoplethysmograph on the finger, these two measures differ only by a constant factor which varies from $S$ to $S$ depending on the S's physical condition, physical dimensions, and age. Thus, for operant conditioning experiments, pulse transit time provides an excellent correlate of blood pressure. The apparatus described here provides a highly accurate measure of pulse transit time and satisfies all six of the criteria presented earlier for an ideal response information device. The apparatus eliminates most of the bulky or complex instruments employed in earlier pulse wave velocity measuring systems (i.e., Sands, 1925; Weltman et al, 1964).

\section{Apparatus}

There are four stages in the measurement of pulse transit time, as depicted in Fig. 1. In the first stage, the electrical signals from the contraction of the heart and the passage of blood through the finger are amplified. In the second stage, the heart beat, represented by the electrocardiogram signal, and the pressure pulse, represented by the photoplethysmogram signal, are detected and shaped for later processing. In the third stage, the temporal interval between the two pulses is measured and compared with the preselected criterion interval. In the fourth stage, the various display and recording functions are performed.

A number of options are available to the $E$ in constructing this system, ranging from constructing the entire apparatus from integrated circuits to assembling the entire apparatus from commercially wired equipment. The integrated circuit option produces a very small, portable, inexpensive device, but requires some amount of construction time and skill. The commercially wired option utilizes equipment already in many operant laboratories, requires very little construction time, but is not portable and is fairly expensive. The authors employed a mixture of integrated circuits and commercially wired equipment. The following description of the instrumentation details the authors' choices, but references are made to the other available options. 
Stage 1. A physiological recorder is used to perform the functions of Stage 1. The EKG is sensed with two electrodes placed on either side of the $S$ 's chest and a centrally placed ground electrode. These electrodes go directly to a preamplifier channel of the recorder (Physiograph Four, E \& M Instrument Co.). The finger pulse is sensed with a photoplethysmograph (Part No. 91-500-70, E \& M Instrument Co.), which is connected to another preamplifier channel of the recorder. The photoplethysmograph (composed of a light bulb, battery, and photocell) photoelectrically measures the volume of blood in the finger. The passage of the pulse wave through the finger vessels produces a proportionate change in the signal from the photoplethysmograph.

For the E who does not want to use a recorder, a small, integrated circuit amplifier for both the EKG and the finger pulse can easily be constructed (Jernstedt \& White, 1974).

Stage 2. Amplifiers $2-\mathrm{I}$ and $2-\mathrm{II}$ are designed to prevent large voltage swings from reaching succeeding stages and to change the polarity of the signals from Stage 1 without inverting them. The circuit for each amplifier, as diagrammed in Fig. 2, is a simple transistor amplifier with a near-unity amplification factor. The input to the amplifier is the sum of the recorder's preamplifier output signal and a constant voltage sufficient to change the pulse signal from a negative to a positive voltage. This part of Stage 2 is not needed if Stage 1 uses a recorder with positive voltage output or the integrated circuit amplifiers described by Jernstedt and White (1974).

Two Schmitt triggers (2-III and 2-IV) form the second part of Stage 2. The turn-on voltage of each trigger circuit may be adjusted empirically by the $E$ so that it is high enough to ignore changes in the EKG or finger signal between pulses and low enough to fire for every pulse.

Since the duration of the EKG and finger pulses is not of interest and since the following logic requires very fast rise times, the two one-shots (2-V and 2-VI) produce 250 -nsec pulses whose onsets coincide with the onset of the R-wave component of the EKG and the finger pulse, respectively.

Stage 3. The outputs from the pulse forming one-shots of Stage 2 are fed to Flip-Flop 3-I. The EKG pulse sets the flip-flop, and the finger pulse resets it so that the output of the flip-flop is logical 1 for a period of time corresponding to the pulse transit time from the heart to the finger. Driver 3-VI follows the flip-flop output and converts it to a voltage and current suitable for the display devices.

The output from One-Shot 2-V also turns on One-Shot 3-II. The duration of One-Shot 3-II is adjusted by the E to correspond to a criterion pulse transit time. Connection of either the 1 output or the 0 output of One-Shot 3-II to AND 3-II will produce a pulse at the output of the AND for each pulse transit time that is either below or above criterion, whichever the $\mathrm{E}$ desires. One-Shot 3-IV and Driver 3-V modify the pulse output from the AND so that it can drive an external display device.

Stage 4. The output devices discussed here are only a suggestion of what may be done with the two output signals obtained from Stage 3. These suggestions provide an example of each of the four major types of output information which the operant $\mathrm{E}$ might wish to employ. All four options employ the signals on Lines 3-a and 3-b as input, have been tested by the authors, and have been used in a series of experiments investigating the conditioning of pulse wave velocity. The following description of Stage 4 discusses the use of this part of the apparatus in conditioning studies.

The first option provides a display for the $S$ of his pulse transit time and whether the time meets the reinforcement criterion. A Tektronix Type 564 oscilloscope is used for the display. Line $3-b$ is connected to Channel 1 of a Tektronix Type 3A72 dual-trace amplifier, and a Tektronix Type 2B67 time base is adjusted to trigger the horizontal sweep at the onset of the signal on Line 3-b. Line 3-a is connected to Channel 2 of the amplifier, and the amplification factor and base line of Channel 2 are adjusted so that a signal on Line 3 -a produces a

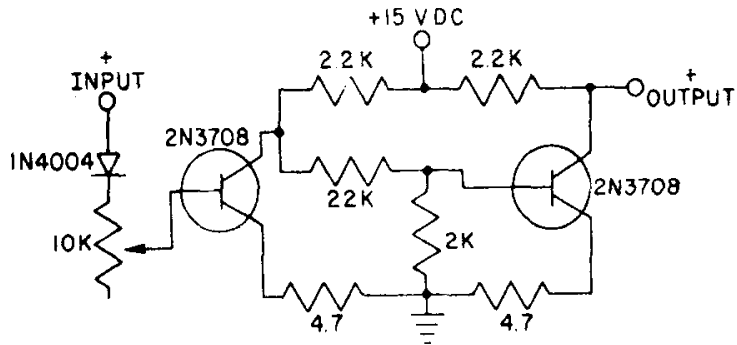

Fig. 2. Circuit diagram of the initial amplifiers (2-I and 2-II) of Stage 2. All resistors are $1 / 2 \mathrm{~W}, 10 \%$.

large vertical line on the screen. Thus, for each heartbeat, the $S$ sees on the screen a horizontal line whose length is proportional to the pulse transit time. For each heartbeat for which the pulse transit time meets the reinforcement criterion, the $S$ sees a vertical band terminate the horizontal line. Using the $5 \mathrm{X}$ magnification factor on the horizontal sweep produces large and . clearly identifiable changes in the display for small changes in pulse transit time.

The second output device provides a discrete reinforcement to the $\mathrm{S}$. The signal on Line 3-a operates a standard electromechanical relay which, in turn, operates a reinforcement delivery apparatus. This additional reinforcement, which is the same as that used by Shapiro et al (1969), consists of a 5-sec display of a Playboy nude slide presented on a screen adjacent to the oscilloscope for every 20 criterion pulse transit times.

The third output device provides a precise, permanent digital record of each pulse transit time. The signal on Line 3-b operates a relay which operates a time interval measurement system. This system records the pulse transit time for each heartbeat. Although the authors use a Hewlett-Packard 2010 data acquisition system, any other time interval measurement device may be connected to the relay operated from Line 3-b.

The fourth output device provides a permanent analog record of the various signals employed in the experiment. The E \& M physiograph mentioned previously is used. Channels 1 and 2 of the recorder are used to record the EKG and the finger pulse signals. Channel 3 is connected to Line $3-\mathrm{a}$ and therefore provides a count of criterion pulse transit times. Channel 4 is connected to a switch and battery, so the $\mathrm{E}$ can mark trial onsets and other relevant information on the recorder record.

Construction. Stages 2 and 3, the logic parts of the apparatus, were constructed from integrated circuits. They could equally as well have been assembled from commercially wired logic modules. The use of integrated circuits allowed the construction of a very inexpensive but highly reliable and compact device. The integrated circuits used were Motorola Type MHTL, which are designed for situations in which high levels of electrical noise are present (such as might be found with a freely moving $\mathbf{S}$ ). All wiring was done with Cinch DIP sockets, into which the integrated circuits were plugged when the wiring was finished. A few other components were needed to set the time durations of the one-shots and to construct the transistor amplifiers, which were not available in integrated circuit form. All components were assembled on a $0.1 \times 0.15 \mathrm{~m}$ piece of perforated circuit board. The use of integrated circuits reduced the construction time to a few hours. Figure 3 contains the complete wiring diagram of Stages 2 and 3.

\section{Operation}

The assembled apparatus was tested by comparing readings taken with it to readings obtained with a sphygmomanometer. Two Ss were used. Each was asked to run in place for $1 \mathrm{~min}$ to provide a raised blood pressure which would decrease as the $S$ rested. Immediately after the $S$ finished running, the pulse transit time apparatus and the sphygmomanometer were attached to him. Readings were then taken of pulse transit time 


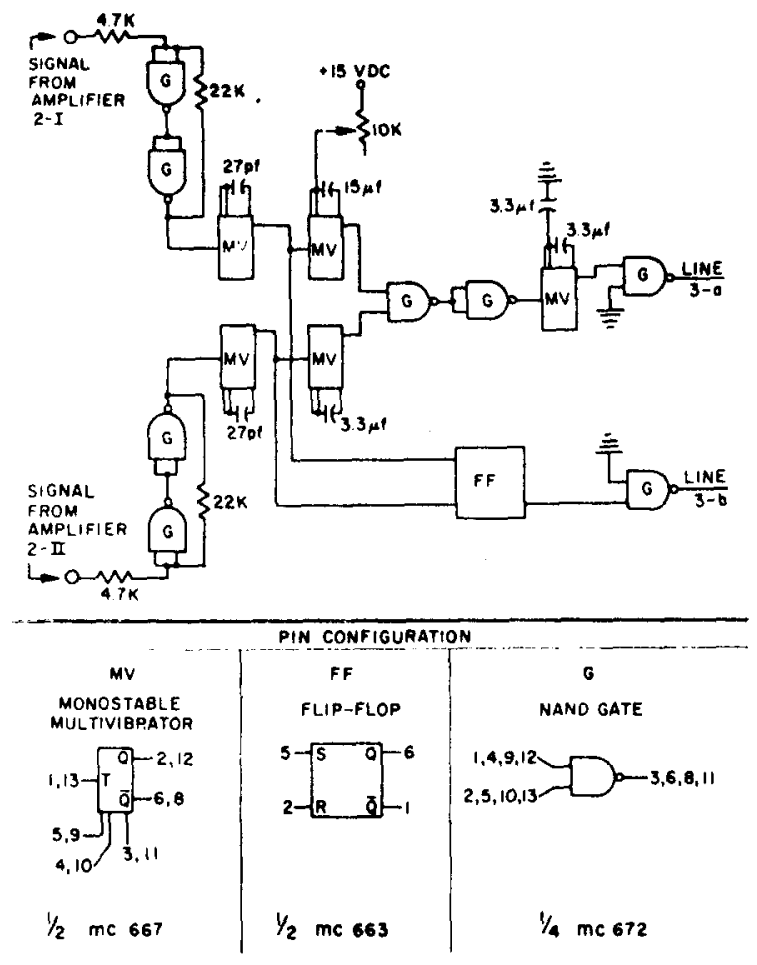

Fig. 3. Circuit diagram of Stages 2 and 3 (with the exception of information contained in Fig. 2). All integrated circuits are Motorola Type MHTL. Resistors are $1 / 2 \mathrm{~W}, 10 \%$. Capacitors in microfarad range are solid tantalum, $20 \%$, and capacitors in picofarad range are mica, $10 \%$.

and blood pressure as the $\mathrm{S}$ recovered from the exercise. The arterial distance between the heart and the finger pulse pickup was estimated with a tape measure, using anatomical landmarks, a.,d was used to calculate pulse wave velocity from pulse transit time.

\section{RESULTS}

The relationship between pulse wave velocity and blood pressure is plotted in Fig. 4. The product moment correlation coefficient between the two measures was 0.97 for $S 1$ and 0.92 for $S 2$.

Although the data will be reported elsewhere, it should be mentioned here that the described procedures were effective in pilot studies in producing Ss who could alter their pulse wave velocity under operant control. Under the procedure described above, and similar to that used by Shapiro et al (1969), two Ss were able to change their pulse wave velocity over a total period of $3 \mathrm{~h}$, each producing a net change in pulse wave velocity of approximately $0.35 \mathrm{~m} / \mathrm{sec}$, which was accompanied by a net change in blood pressure of approximately $10 \mathrm{~mm} \mathrm{Hg}$. Heart rate changes were not correlated with either blood pressure or pulse wave velocity during the conditioning period.

These data are not included as proof of the correlation between pulse wave velocity and sphygmomanometrically measured blood pressure, since the literature cited previously is relatively clear on this matter. Rather, these data are presented in support of the described procedures and instrumentation.

\section{DISCUSSION}

According to available evidence, pulse wave velocity should accurately reflect conventionally measured blood pressure. The obtained high correlation between sphygmomanometrically measured blood pressure and pulse wave velocity was even higher than that reported by Sands (1925), presumably because of the improved apparatus for measuring pulse wave velocity. The error in measurement with sphygmomanometers reported by Smith and Bickley (1964) suggests that the obtaining of a very high correlation between pulse wave velocity and blood pressure is limited by errors in the blood pressure measurement system and not by errors in pulse wave velocity measurement or a lack of high correlation between pulse wave velocity and blood pressure. That the two measures are highly correlated under all conditions should not be concluded, since Sands (1925) and King (1950) indicated that various pathological conditions and other long-term changes in the body will affect the correlations.

Williams and Williams (1965) suggested that pulse wave velocity measures taken from heart to finger were less reliable than measures taken along the upper arm only. This suggestion was not followed here because the instrumentation is far easier to use and more noise-free with a finger photoplethysmogram and, most importantly, because the measure used for conditioning is pulse transit time. Since pulse wave velocity increases as arterial diameter increases, pulse transit time along lower arm and finger is a relatively small component of overall pulse transit time from heart to finger. Thus, any lack of reliability in the measurement of pulse wave velocity itself is offset by the increased reliability of the finger transducer. However, investigators for whom upper arm plethysmograms are more desirable can substitute the appropriate transducer for the finger photoplethysmogram used in this study. No major changes in instrumentation are required.

One might ask if changes in the pulse transit time measure may be produced by artifacts such as changes in the waveform of the EKG or finger pulse. The rise time of the EKG and finger pulses is extremely rapid when compared to the time between

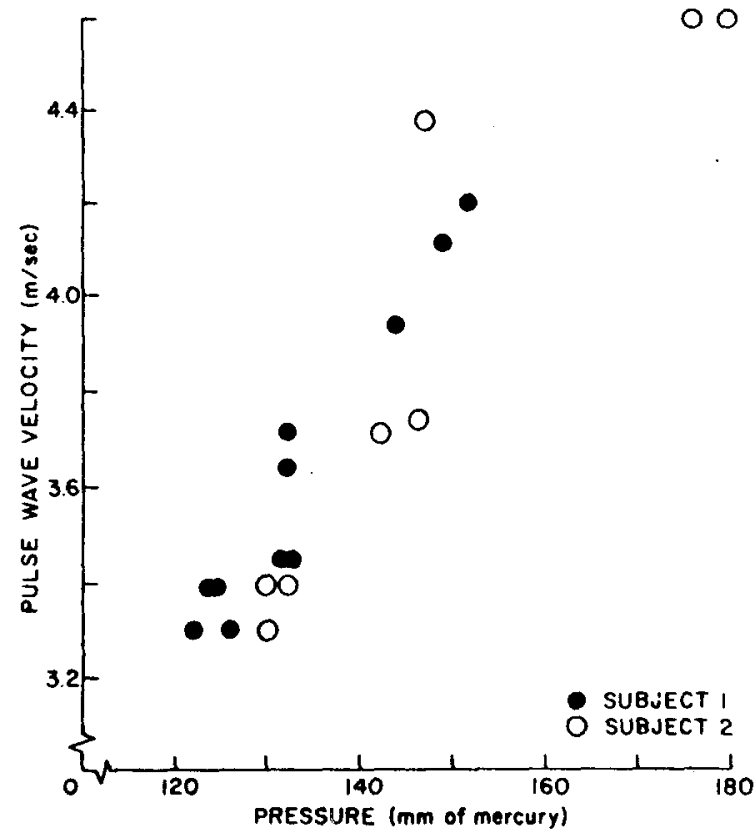

Fig. 4. Relationship between pulse wave velocity and sphygmomanometric systolic blood pressure. 
these pulses, and the Schmitt triggers fire on the onset (rather than the peak level) of the pulses. Therefore, changes in the waveform of the two pulses should produce insignificant changes in the measured pulse transit time.

If the $\mathrm{S}$ moves while the photoplethysmograph is in place, there may be large fluctuations in output due to movement artifacts. Such artifacts associated with the finger pulse signal may cause firing of the Schmitt trigger at times other than when a pulse is being detected; this is the most serious problem associated with the apparatus. The solution is to restrain the S's hand in a frame (as was done with the authors' apparatus) or add a circuit to discriminate between the pressure pulse signals and movement artifacts. Winter and Trenholm (1969) describe a circuit to filter movement artifacts from EKG signals which works with Ss engaged in vigorous exercise. This circuit is equally useful for filtering movement artifacts from the photoplethysmograph signal. The investigator for whom restraint of the $S$ is undesirable may replace Schmitt Trigger 2-V with the circuit described by Winter and Trenholm (1969). The amplifiers described by Jernstedt and White (1974) employ such a filter circuit, so that using these amplifiers in place of the recorder will attenuate movement artifact problems.

In normal operation of a finger photoplethysmograph, there arise occasions when the finger pulse is lost. For example, vasoconstriction during stress may reduce the finger pulse below the trigger level of the Schmitt trigger. With the apparatus described here, occurrences of lost finger pulses, for whatever reason, never eliminated more than 1 of 10 successive pulses in the worst case. Though they may be disturbing, these problems are also encountered with many of the methods of blood pressure measurement in current use.

As Weltman et al (1964) and Williams and Williams (1965) suggest, pulse wave velocity may, in fact, be a better measure of cardiovascular performance than conventionally measured blood pressure. The use of blood pressure changes as a response in operant conditioning experiments and the use of pulse wave velocity as an indicator of blood pressure both seem to have been limited by instrumentation problems. The present procedure and its associated apparatus provide an accurate and convenient solution to these problems.

\section{REFERENCES}

Brener, J., \& Kleinman, R. A. Learned control of decreases in systolic blood pressure. Nature, 1970, 226, 1063-1064.

Jernstedt, G. C., \& White, W. F. Cardiovascular response measures with simple integrated circuit amplifiers. Psychophysiology, 1974, 11, 211-215.

King, A. L. Circulatory system: Arterial pulse; wave velocity. In O. Glasser (Ed.), Medical physics. Vol. 2. Chicago: Year Book Publishers, 1950. Pp. 188-191.

Miller, N. E., \& Banuazizi, A. Instrumental learning by curarized rats of a specific visceral response, intestinal or cardiac. Journal of Comparative \& Physiological Psychology, 1968, 65, $1-7$.

Sands, J. Studies in pulse wave velocity: III. Pulse wave velocity in pathological conditions. American Journal of Physiology. $1925,71,519-533$

Shapiro, D., Tursky, B., Gershon, E., \& Stern, M. Effects of feedback and reinforcement on the control of human systolic blood pressure. Science, $1969,163,588-590$.

Shapiro, D., Tursky, B., \& Schwartz, G. E. Control of blood pressure in man by operant conditioning. Supplement I to Circulation Research, 1970a, 26 \& 27, 27-32.

Shapiro, Tursky, B., \& Schwartz, G. E. Differentiation of heart re: and systolic blood pressure in man by operant conditioning. Psychosomatic Medicine, 1970b, 32, 417-423

Smith, C. R., \& Bickley, W. H. The measurement of blood pressure in the human body. Technology Survey No. NASA SP-5006. Washington, D.C: National Aeronautics \& Space Administration, 1964.

Weltman, G., Sullivan, G., \& Bredon, D. The continuous measurement of arterial pulse wave velocity. Medical Electronics \& Biological Engineering, 1964, 2, 145-154.

Williams, J. G. L., \& Williams, B. Arterial pulse wave velocity as a psychophysiological measure. Psychosomatic Medicine, 1965, $27,408-414$.

Winter, D. A., \& Trenholm, B. G. Reliable triggering for exercise electrocardiograms. IEEE Transactions on Biomedical Engineering, 1969, BME-16, 75-79.

(Received for publication March 8, 1974; revision received May $7,1974$. 